\title{
Detection of a possible case of Pulmonary Tumour Thromboembolism by endobronchial ultrasound
}

\author{
S.S. Dhillon'1, I.G. Berim², N. Khushalani1, J. Gannon³
}

A 52-year-old woman with a history of recurrent melanoma underwent surveillance CT scan during adjuvant high-dose interferon therapy revealing a new right posterior thorax lesion. Eighteen months prior, she had developed pulmonary thromboembolic disease and an inferior vena cava filter was placed along with anticoagulation therapy with subsequent radiographs revealing complete resolution of the pulmonary emboli. 18F-FDG PET-CT was performed for further evaluation, which showed the posterior thorax lesion to be intensely FDG avid with a maximum SUV of 58.9. Another intensely FDG-avid area was seen in the medial right upper lung extending into the adjacent supra-hilar and azygous region with a maximum SUV of 30.3 (figure 1) but the corresponding CT images did not reveal any lymphadenopathy or anatomical abnormality.

An endobronchial ultrasound (EBUS) was performed as a diagnostic procedure to further evaluate these abnormal radiological findings. A thorough evaluation of right paratracheal and hilar region by EBUS revealed a single $0.5 \mathrm{~cm}$ lymph node with aspiration demonstrating lymphoid tissue with no evidence of any malignant cells. EBUS also revealed a hyperechoic lesion in the right main pulmonary artery extending to the upper lobe branch (figure 2).

CT angiography performed the same day demonstrated a large filling defect in the right main pulmonary artery extending into and completely occluding the right upper lobe pulmonary artery branch (figure 3 ). There was no evidence of deep venous thrombosis in the abdominal and lower extremity veins. Comparison to the PET-CT imaging showed this lesion to correspond to the site of FDG-avidity and this was felt to represent a combination of tumour thrombus and bland thrombus due to the fact that SUV value was very high. Anticoagu- lation was initiated along with plans for additional anti-neoplastic therapy.

EBUS has become an invaluable safe and minimally invasive modality for sampling mediastinal lymph nodes. The major thoracic vessels serve as important landmarks for recognising lymph node stations in the new lung cancer staging system and should be carefully evaluated during EBUS procedures. EBUS has recently been shown to be able to recognise emboli in pulmonary vessels in patients with confirmed pulmonary embolism [1]. This case demonstrates a potential role of EBUS in the evaluation of PET positive areas in the vicinity of thoracic vessels where a clear anatomical abnormality or lymphadenopathy is not identified on the CT.

CT scan is an excellent test to evaluate mediastinal and hilar lymphadenopathy but occasionally suffers from pitfalls like volume averaging artifact making it hard to evaluate subcarinal and hilar lymph nodes and beam hardening artifact from contrast in superior vena cava making it difficult to assess the right paratracheal area. Recognising small lymph nodes in the vicinity of major hilar vessels can be challenging. Okamoto and colleagues performed radial EBUS to evaluate mediastinal and hilar lymph node metastasis in lung cancer and found hilar lymph nodes in 11 out of 27 patients in whom a clear lymph node was not identified on the CT imaging [2]. It is therefore reasonable to evaluate this area with EBUS even if the corresponding CT does not show any lymphadenopathy and lymph node metastasis is suspected.

During transbronchial needle aspirations, major thoracic vessels can be inadvertently punctured and the reported incidence of major bleeding is apparently low (however these patients are not routinely imaged after the procedure and the exact incidence of bleeding is not known). A case has been described where a
Monaldi Arch Chest Dis 2012; 77: 1, 40-42

Keywords:

Endobronchial

Ultrasound, EBUS,

Tumor embolism,

Melanoma.

1 Department of Medicine, Roswell Park Cancer Institute, Buffalo, NY,

2 Department

of Pulmonary and Critical Care Medicine State University of New York at Buffalo, NY,

3 Department of

Radiology, Roswell Park Cancer Institute, Buffalo, NY, U.S.A.

Correspondence: Samjot Singh Dhillon. MD, Department of Medicine - Thoracic Oncology, Roswell Park Cancer Institute, Elm and Carlton Streets, Buffalo, NY 14263, U.S.A.; e-mail: samjot.dhillon@ roswellpark.org 


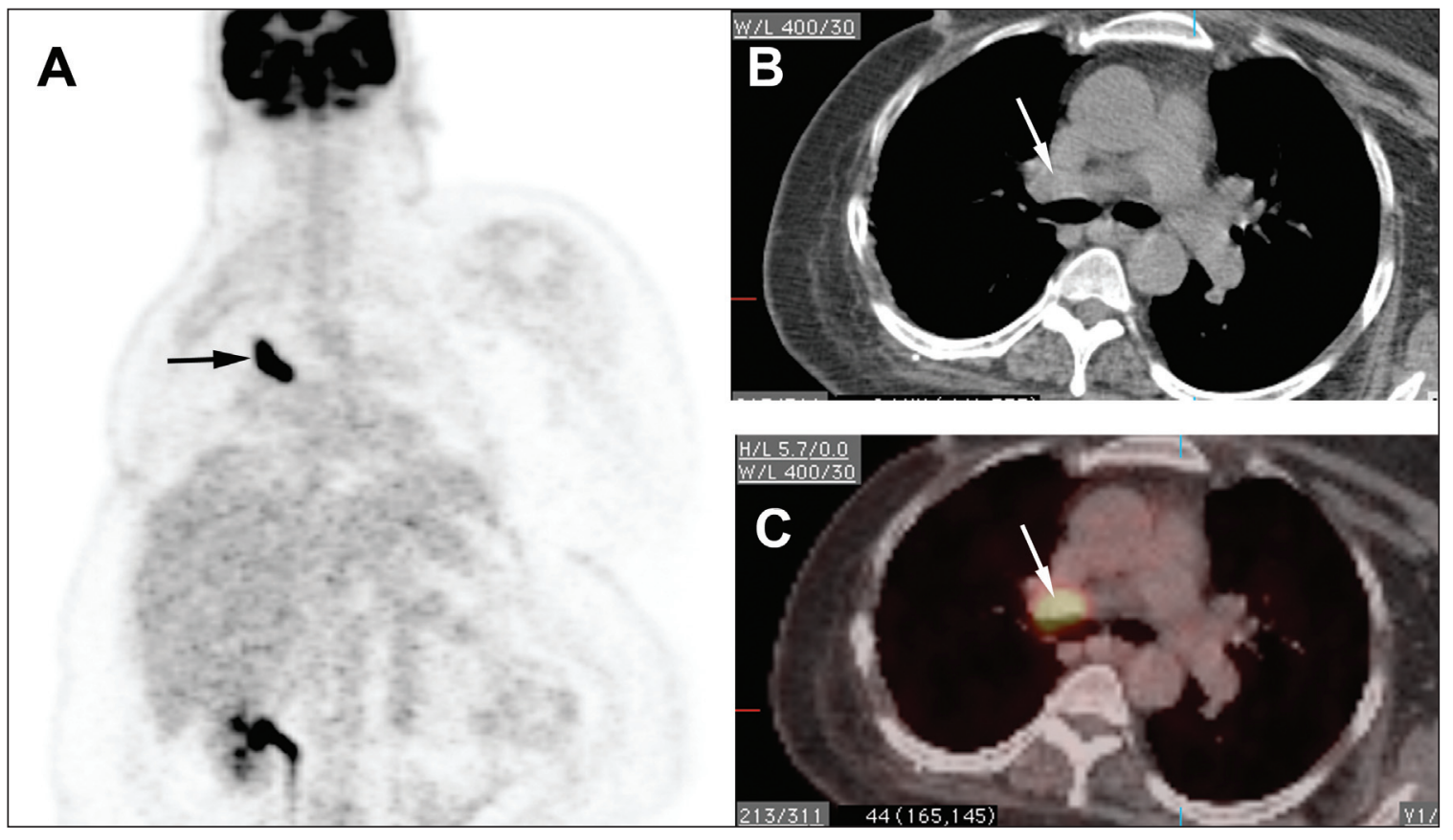

left hilar node was safely accessed by traversing the EBUS needle through the left pulmonary artery [3] and another case of metastatic thyroid tumour embolism has also been reported after successful EBUS guided aspiration of non-resolving pulmonary thrombus [4]. On the contrary, some case reports have described complications like mediastinal bleeding, hematoma and mediastinitis after EBUS.
To date no large study has shown the safety of transbronchial puncture of major thoracic vessels and thus such an approach should be pursued with caution and only if absolutely necessary.

A few small studies and several case reports have suggested some focal FDG uptake in bland pulmonary thromboembolism but generally the SUV is very low and almost sim-
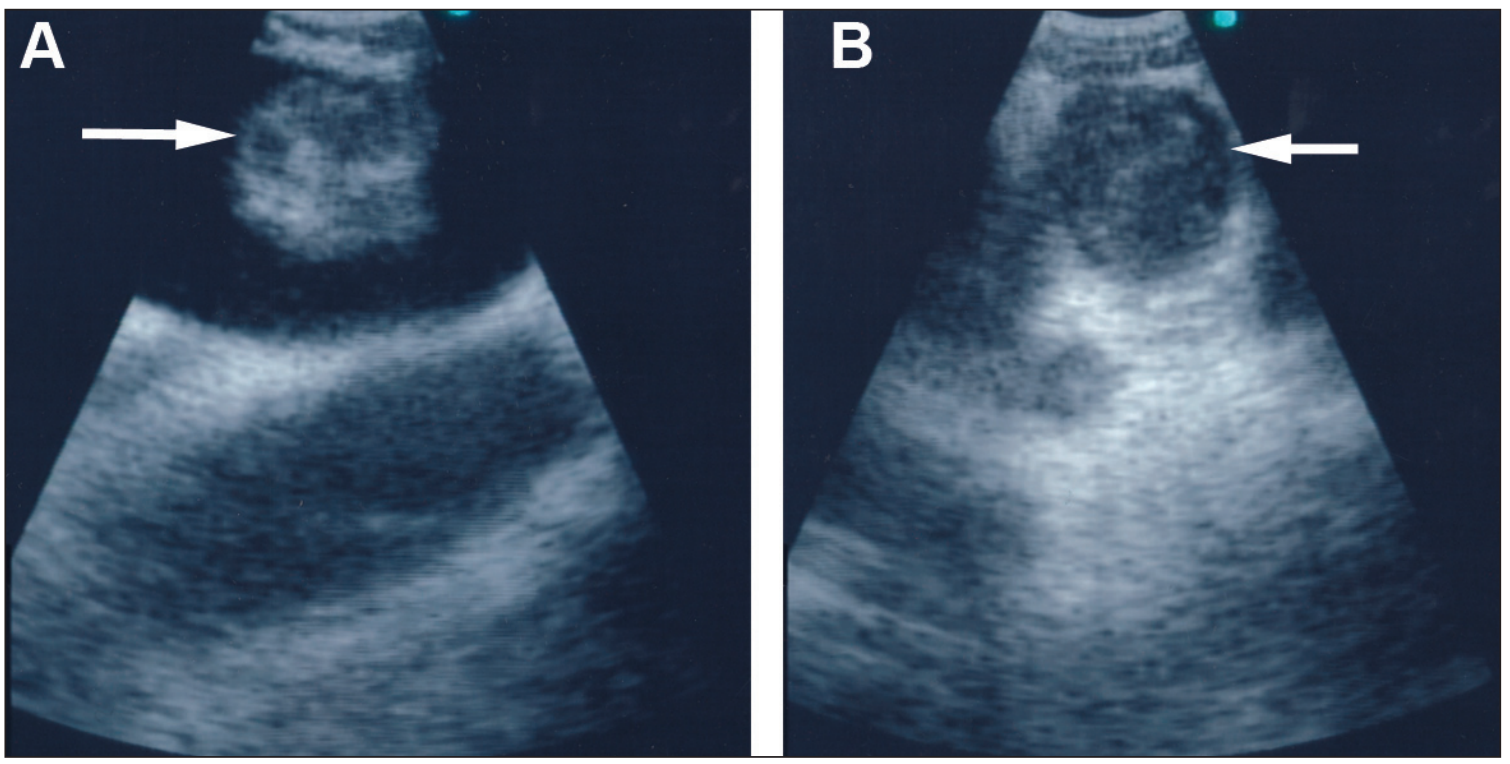

Fig. 1. - Coronal PET image showing significantly increased FDG uptake in right paratracheal and hilar area in a curvilinear fashion (Panel A, black arrow); the fused images showing intense FDG uptake in this area (Panel C, white arrow) with corresponding axial CT images not showing any lymph node (Panel B, white arrow)

Figure 2. - EBUS images showing hyperechoic lesion in right main (Panel A, white arrow) and right upper lobe branch (Panel B, white arrow) pulmonary artery. 


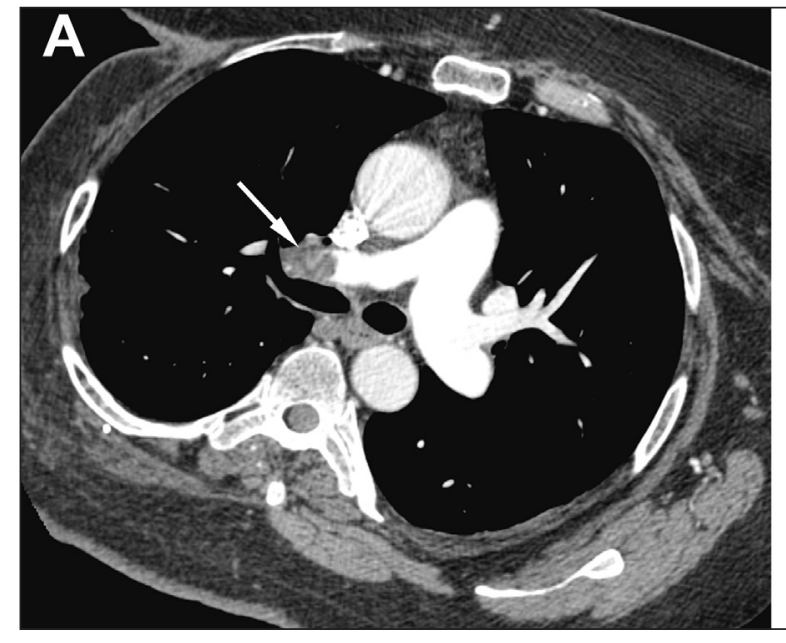

ilar to or slightly above the background signal. In a study by Wittram and Scott the mean SUV of acute pulmonary embolism was quite low (1.65 \pm 0.61 ; range $0.45-3.03)$ [5]. Although, we did not obtain histological diagnosis in our patient, we believe that the extremely high SUV was convincing of a clinical diagnosis of Pulmonary Tumour Thromboembolism.

This case was unusual as the PET showed a curvilinear area of significant FDG uptake in the right paratracheal and hilar region without identification of any lymph nodes. The EBUS helped clarify this ambiguous PET-CT finding. Embolism to the pulmonary artery should be suspected if curvilinear area of increased FDG uptake is seen over or close to the location of pulmonary vessels, especially if a lymph node is not definitively seen [5].

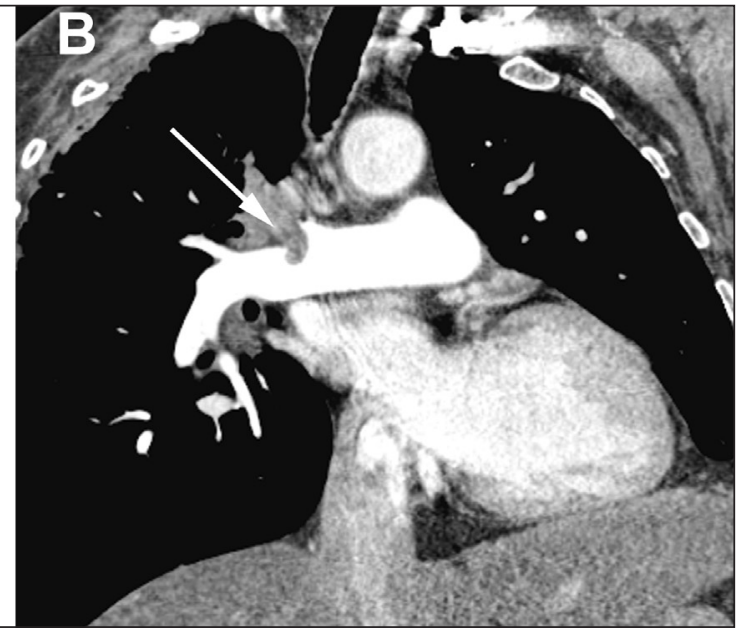

Figure 3. - CT pulmonary angiogram with axial (Panel A) and coronal (Panel B) sections demonstrating thrombus in right main pulmonary artery (Panel A, white arrow) and almost completely occluding right upper lobe pulmonary artery branch (Panel B, white arrow).

1. Aumiller J, Herth FJ, Krasnik M, et al. Endobronchial ultrasound for detecting central pulmonary emboli: a pilot study. Respiration 2009; 77: 298-302.

2. Okamoto H, Watanabe K, Nagatomo A, et al. Endobronchial ultrasonography for mediastinal and hilar lymph node metastases of lung cancer. Chest 2002; 121: 1498-1506.

3. Vincent B, Huggins JT, Doelken P, et al. Successful real-time endobronchial ultrasound-guided transbronchial needle aspiration of a hilar lung mass obtained by traversing the pulmonary artery. $J$ Thorac Oncol 2006; 1: 362-364.

4. Blanc AL, Jardin C, Faivre JB, et al. Pulmonary artery tumour-embolism diagnosed by endobronchial ultrasound-guided transbronchial needle aspiration. Eur Repir J 2011; 38: 477-479.

5. Wittram C, Scott JA. 18F-FDG PET of pulmonary embolism. AJR 2007; 189: 171-176. 